skin relaxation time correlated negatively, with mRTSS $(P<0.05$ for all correlations). Functional disability, which was measured using an 11-item, validated questionnaire, correlated more closely with Vesmeter ${ }^{\circledR}$ measured skin hardness $(r=0.643, P=0.0021)$ than with mRTSS $(r=0.517, P=0.0193)$. Mean intraobserver and interobserver variability of skin hardness values was $7.63 \%$ and $19.76 \%$, respectively (lower than reported variabilities for mRTSS).

The authors conclude that the Vesmeter ${ }^{\circledR}$ provides reliable quantification of skin hardness and might be useful in clinical trials of patients who have systemic sclerosis with skin involvement.

Original article Kuwahara Y et al. (2008) Quantification of hardness, elasticity and viscosity of the skin of patients with systemic sclerosis using a novel sensing device (Vesmeter): a proposal for a new outcome measurement procedure. Rheumatology (Oxford) [doi:10.1093/rheumatology/ken145]

\section{Gout: the HAQ DI is a reliable measure of physical function}

The Health Assessment Questionnaire Disability Index (HAQ DI) is widely used for the assessment of rheumatic disease, and severe gout has been associated with high HAQ DI scores. A Mexican, multicenter, cohort study has assessed the validity of the HAQ DI for the evaluation of gout.

Álvarez-Hernández et al. evaluated the $\mathrm{HAQ}$ $\mathrm{DI}$ as a measure of function in 206 patients (199 men) with gout. Mean patient age was 56.3 years, and mean disease duration was 9.3 years. In all, $52.4 \%$ of patients had joint pain, $22.8 \%$ had swelling, $32.5 \%$ had reduced mobility and $36.9 \%$ had tophi. The HAQ DI correlated well with clinical symptoms, and had convergent validity with the Short Form 36, with particularly good correlations in physical functioning, bodily pain and the physical component summary. The instrument was able to discriminate between patient subgroups on the basis of clinical variables. Test-retest-reliability analysis produced an intraclass correlation coefficient of 0.76 and an overall Cronbach's $\alpha$ (internal consistency) coefficient of 0.94 . Sensitivity-to-change analysis included 167 patients who retook the HAQ DI 6 months after the initial assessment. HAQ DI scores correlated with improvement or deterioration in patient symptoms; the overall effect size was
0.62 and Guyatt's responsiveness index score was 1.91.

The authors conclude that the HAQ DI is a valid and reliable instrument for measuring physical function in patients with gout.

Original article Álvarez-Hernández E et al. (2008)

Validation of the Health Assessment Questionnaire Disability Index in patients with gout. Arthritis Rheum 59: 665-669

\section{New tool for assessing enthesitis in patients with psoriatic arthritis}

Enthesitis is thought to underlie most manifestations of psoriatic arthritis. While there are several tools that assess enthesitis in ankylosing spondylitis, development of a validated measure that is specific to psoriatic arthritis has received little research attention. Healy and Helliwell evaluated the currently available tools, and developed a new instrument-the Leeds Enthesitis Index (LEI) - for use in patients with psoriatic arthritis.

This open-label, observational trial included 28 patients (mean age 46.5 years) with active psoriatic arthritis who started treatment with methotrexate $(n=19)$, leflunomide $(n=4)$, etanercept $(n=4)$ or hydroxychloroquine $(n=1)$. Patients were assessed 2 weeks, 1 month, 3 months and 6 months after initiation of treatment, with the following evaluations: Mander Enthesitis Index (MEI), Maastricht Ankylosing Spondylitis Enthesitis Score, Gladman Index, Psoriasis Area and Severity Index, 78-joint count and the Health Assessment Questionnaire.

The LEI was developed by applying an iterative data-reduction process to MEI scores. The LEl includes six examination points (left and right lateral epicondyles, medial femoral condyles and Achilles tendon insertions). All enthesitis indices correlated strongly with each other and with measures of disease activity, and all showed a floor effect (i.e. a score of 0 when the MEI $>0$ ), although this effect was minimal for the LEI.

The LEI seems to be a robust and reliable tool for assessing enthesitis in patients with psoriatic arthritis; the authors suggest that it should be used in future clinical trials.

Original article Healy PJ and Helliwell PS (2008) Measuring clinical enthesitis in psoriatic arthritis: assessment of existing measures and development of an instrument specific to psoriatic arthritis. Arthritis Rheum 59: 686-691 